UCRL-MA- 108598

\title{
CAST2D: a finite element computer code for casting process modeling
}

\author{
Arthur B. Shapiro
}

Lawrence Livermore National Laboratory

John O. Hallquist

Livermore Software Technology Corp.

October 1991

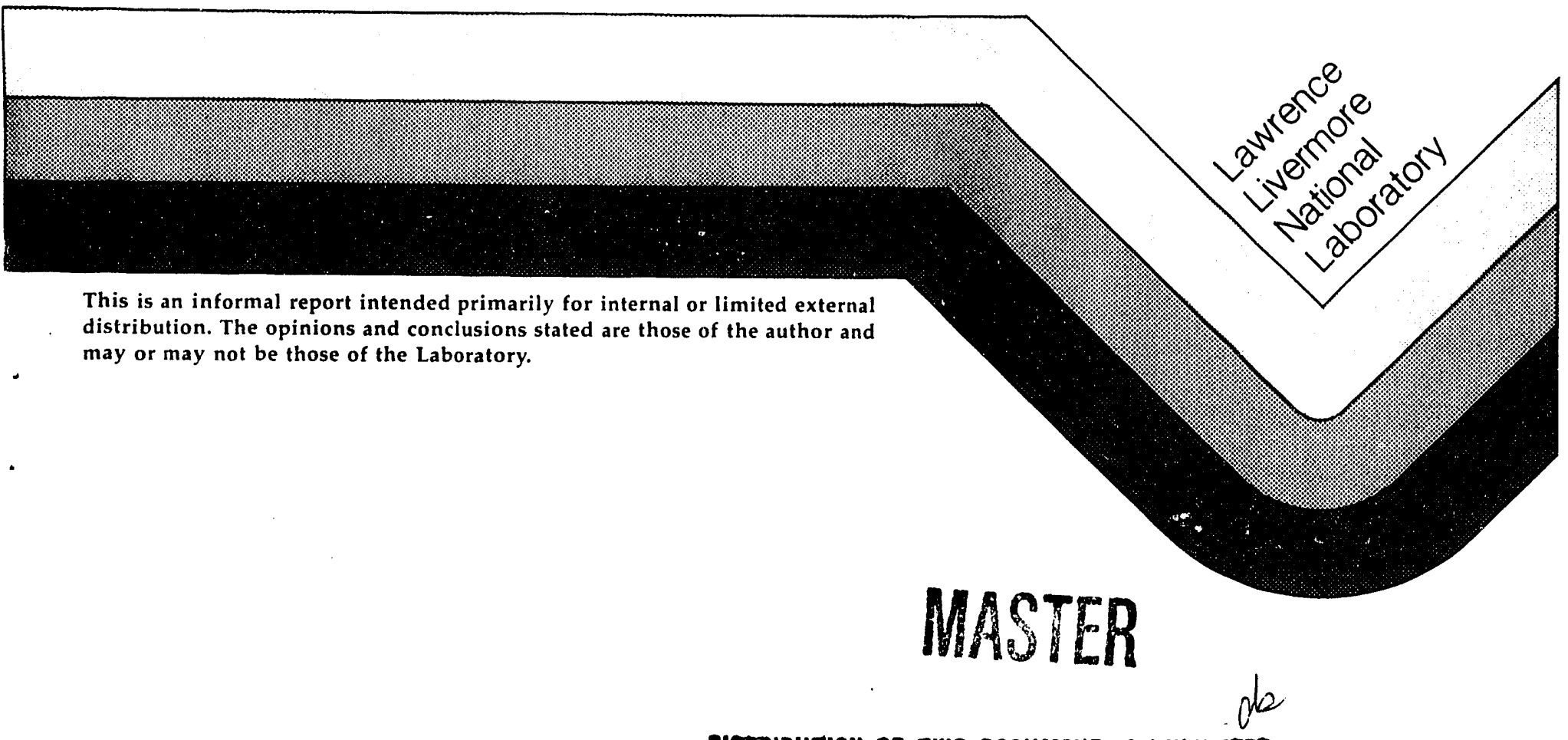




\section{DISCLAIMER}

This document was prepared as an acccount of work sponsored by an agency of the United States Government. Neither the United States Goveriment nor the University of California nor any of their employees, makes any warranty, express or implied, or assumes any legal liability or responsibility for the accuracy, completeness, or usefulness of any information, apparatus, product, or process disclosed, or represents that its use would not infringe privately own rights. Reference herein to any specific commercial products, process, or service by trade name, trademark, manufacturer, or otherwise, does not necessarily constitute or imply its endorsement, recommendation, or favoring by the United States Government or the University of California. The views and opinions of authors expressed herein do not necessarily state or reflect those of the United States Government or the University of California, and shall not be used for advertising or product endorsement purposes.

This report has been reproduced directly from the best available copy.

Available to DOE and DOE contractors from the Office of Scientific and Technical Information P.O. Box 62, Oak Ridge, TN 37831

Prices available from (615) 576-8401, FTS 626-8401

Available to the public from the

National Technical Information Service

U.S. Department of Commerce

5285 Port Royal Rd.

Springfield, VA 22161

Work performed under the auspices of the U.S. Department of Energy by Lawrence Livermore National Laboratory under Contract W'-7405-Eng-48. 


\title{
CAST2D: a finite element computer code for casting process modeling
}

\author{
Arthur B. Shapiro \\ Lawrence Livermore National Laboratory \\ John O. Hallquist \\ Livermore Software Technology Corp.
}

October 1991

DRAFT - this draft manual is issued so that users can begin using the code. Significant additions will be made in the future.

Lawrence Livermore National Laboratory

7000 East Ave.

Livermore, CA 94550

phone: $415-422-5066$

fax: $415-422-1370$ 


\section{Table of Contents}

1 Introduction 1

2 Theory 3

2.1 Background 3

2.2 Methodology 4

3 Input and Execution 7

3.1 Pre-processing 7

3.2 Special input notes 7

3.2.1 NIKE2D input data 7

3.2.2 TOPAZ2D input file 7

3.3 Units 8

3.4 Post-processing 8

3.5 Execution 8

3.6 Interactive Controls 9

References 11 


\section{Introduction}

CAST2D is a coupled thermal-stress finite element computer code for casting process modeling. This code can be used to predict the final shape and stress state of cast parts. CAST2D couples the heat transfer code TOPAZ2D [1] and solid mechanics code NIKE2D [2]. CAST2D has the following features in addition to all the features contained in the TOPAZ2D and NIKE2D codes:

- A general purpose thermal-mechanical interface algorithm (i.e., slide line) that calculates the thermal contact resistance across the part-mold interface as a function of interface pressure and gap opening.

- A new phase change algorithm, the delta function method, that is a robust method for materials undergoing isothermal phase change.

- A constitutive model that transitions between fluid behavior and solid behavior, and accounts for material volume change on phase change.

- A modified plot file data base that allows plotting of thermal variables (e.g., temperature, heat flux) on the deformed geometry.

Although the code is specialized for casting modeling, it can be used for other thermal stress problems (e.g., metal forming).

CAST2D is a code in development. The first three features mentioned above are current research areas. The numerical implementation of these features will be refined over the next year and validated against experimental data. An additional research area is to investigate the coupling of CAST2D with a free surface fluid dynamics code (e.g., ProCAST [3]) so that the entire casting process can be modeled from mold filling to final cast shape.

CAST2D is the result of the efforts of several people working in separate fields. John Hallquist was the originator and developer of the NIKE2D code between 1978-1988. In 1988, Dr. Hallquist founded Livermore Software Technology Corporation where he has continued development of LS-NIKE2D. Arthur Shapiro is the originator and current developer of the TOPAZ2D code. Peter Raboin has made contributions in constitutive modeling to transition between fluid and solid behavior. 


\section{Theory}

\subsection{Background}

TOPAZ2D is a two dimensional implicit finite element computer code for heat transfer analysis. TOPAZ2D can be used to solve for the steady state or transient temperature field on two dimensional planar or axisymmetric geometries. Material properties may be temperature dependent and either isotropic or orthotropic. A variety of time and temperature dependent boundary conditions can be specified including temperature, flux, convection, and radiation. Time or temperature dependent internal heat generation can be defined locally by element or globally by material. TOPAZ2D can solve problems of diffuse and specular band radiation in an enclosure coupled with conduction in the material surrounding the enclosure. Additional features include thermally controlled reactive chemical mixtures, thermal contact resistance across an interface, bulk fluid flow, phase change, and energy balances.

NIKE2D is an implicit, static and dynamic, finite-deformation, finite element computer code for axisymmetric, plane strain, and plane stress problems in solid mechanics. A variety of loading conditions can be handled including traction boundary conditions, displacement boundary conditions, concentrated nodal point loads, body force loads due to base acceleration, and body force loads due to spinning. Slidelines with interface friction are available. Elastic, elastic-plastic, thermal-elastic-plastic, linear visco-elastic, elastic-creep, and strain rate dependent material models are implemented. Nearly incompressible behavior that arises in plasticity problems and elasticity problems with Poisson's ratio approaching 0.5 (e.g., fluids) is accounted for in the element formulation to preclude mesh lock-ups and associated anomalous stress states.

TOPAZ2D and NIKE2D can be used to solve uncoupled thermal-stress problems. First, TOPAZ2D is used to calculate the temperature field at each time step. The temperature-time data at each step is written into a file. Then, NIKE2D reads this temperature-time file and performs the thermal-stress calculations. This uncoupled thermal-stress method is acceptable for problems with small deformation and when no movement between parts occurs. However, for casting and metal forming problems a coupled formulation must be used. In 1984, Gerhard [4] developed a master 
program that would control the execution of TOPAZ2D and NIKE2D for a coupled-thermal stress analysis. This master program would first run TOPAZ2D for a thermal calculation step and then run NIKE2D for a mechanical calculation step. This is referred to as the staggered step approach. Information, such as temperature and current geometry, was passed between TOPAZ2D and NIKE2D by reading and writing files. This resulted in very slow execution. In 1988, Shapiro and Hallquist merged TOPAZ2D and NIKE2D into one source code. Now, information required for a coupled solution (e.g., temperature, geometry) was passed in common blocks resulting in faster execution speeds. This coupled code was used for metal forming applications [5]. CAST2D is an extension of this coupled code incorporating algorithms required for casting process modelling.

\subsection{Methodology}

The TOPAZ2D [1] and NIKE2D [2] user manuals should be referred to for an in-depth discussion of the mathematical theory behind these codes. We will briefly outline here the approach in coupling the two computer programs. The momentum and energy balance laws are

$$
\begin{gathered}
\nabla \cdot \sigma+b=\rho a \\
\sigma \cdot \nabla v-\nabla \cdot q+r=\dot{E}
\end{gathered}
$$

where $\mathbf{v}$ is the particle velocity, $\mathbf{a}$ is the particle acceleration, $\sigma$ is the Cauchy stress tensor, $q$ is the heat flux vector per current area, $\rho$ is the current density, $E$ is the internal energy, and $\nabla$ is the divergence operator per current configuration. $\nabla v$ is the velocity gradient, $b$ is the body force, and $r$ is the internal heat generation. The time derivatives are material, and thus simple partials for a Lagrangian frame where coordinates are assigned to material points.

The heat flux vector, $q$, for conduction of heat in an anisotropic solid is

$$
q=-K \nabla \theta
$$

where $\mathrm{K}$ is the thermal conductivity tensor and $\theta$ is the temperature. The energy balance, Eq. 2 , is then expressed as

$$
\sigma \cdot \nabla v+\nabla \cdot K \nabla \theta+r=\dot{E}
$$

subject to the mixed boundary condition

$$
-K \nabla \theta \cdot n=\alpha \theta-\beta
$$

This boundary condition can represent surface heat flux, convection, or radiation depending on the definition of $\alpha$ and $\beta$. 
The energy and momentum equations with energy dependent mechanical material models must be solved simultaneously in a fully coupled thermal-mechanical code. To achieve this, we use operator splitting. The energy balance is first solved, including the heat due to the stress term. The updated temperatures are then used in the constitutive evaluation as we solve the momentum balance. We iterate for convergence and then proceed to the next step. 


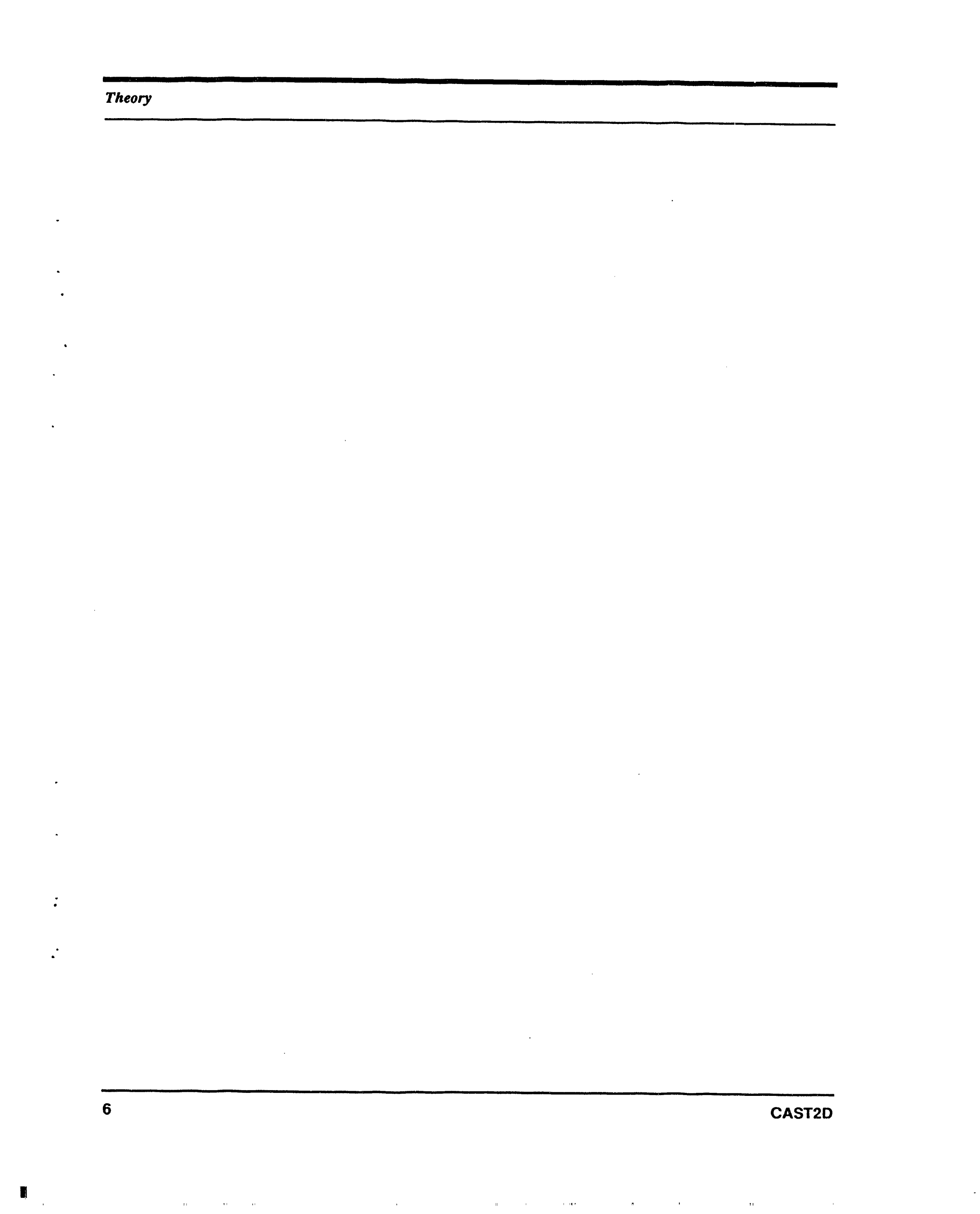




\section{Input and Execution}

\subsection{Pre-processing}

CAST2D requires a combined input file consisting of NIKE2D input data describing the mechanical problem followed by TOPAZ2D input data describing the thermal problem. The interactive pre-processor MAZE [6] can be used to create the mesh, define boundary conditions, material properties, and control parameters for both input files.

\subsection{Special input notes}

\subsubsection{NIKE2D Input data}

- the time step size and ending time must be the same as that in TOPAZ2D

- use oniy slideline type 3 - sliding with voids

- use only material type 4: thermal-elastic-plastic

- set body force loads to base acceleration in z-direction $=1$ (control card 2, columns 31-35) define a load curve for body force load = gravitational acceleration

- set analysis type to dynamic $=1$ (control card 2, columns 41-45)

- set initial condition parameter $=1$ (control card 2, columns 61-65) set initial velocities $=0$.

- set thermal effects option $=0$ (control card 3, columns 11-15)

- set initial temperature flag $=0$ (control card 3, columns 56-60)

- set internal energy dump option $=2$ (control card 3, columns 71-75)

\subsubsection{TOPAZ2D input file}

- the time step size and ending time must be the same as that in NIKE2D

- slidelines:
1) set the thermal contact resistance $=1.0 \mathrm{e}-08 \mathrm{~m}^{2} \mathrm{~K} / \mathrm{W}$ 
2) add the following line containing the variables AIRK and SIGGAP (2E10.0 format) at the beginning of the slide line data.

AIRK = air conductivity across gap (e.g., $0.02 \mathrm{~W} / \mathrm{m} \mathrm{K} \mathrm{s}$ )

SIGGAP = Stefan Boltzmann constant (e.g., 5.67e-08 W/m $\mathrm{m}^{2} \mathrm{~K}^{4}$ )

\subsection{Units}

Any consistent set of units can be used. However, we suggest using SI units to avoid conversion errors between mechanical and thermal units. In SI units the mechanical equivalent of heat is $1 \mathrm{~J} /$ Nm.

\subsection{Post-processing}

CAST2D creates 2 plot files: (1) a NIKE2D output file describing the mechanical response, and (2) a TOPAZ2D output file describing the thermal response. The interactive post-processor ORION [7] can be used to produce graphical output of CAST2D results. Contours and time histories of temperature, heat flux, strain measures, and stress components can be plotted. Note that the TOPAZ2D type output file generated by CAST2D contains deformation information so that ORION can plot temperature and flux contours and the deformed geometry.

\subsection{Execution}

First obtain CAST2D from a take directory by typing

\section{xp read $.806563 /$ cast $2 d$}

To run CAST2D, from the command line enter

\section{cast $2 d i=$ inf $o=$ outf $g=$ mplot $p=t p l o t ~ v=v f f d=d p f r=r t f$}

where

- inf=text input file containing control information, material properties, node point coordinates, element connectivity, and boundary condition specifications.

- outf=text output file containing the input data and output data at selected time steps. The default root name is "c $2 \mathrm{hsp}$ ".

- mplot=binary output plot file containing the mechanical response results that can be graphically displayed using the post processor ORION [7]. The default root name is "m2plot".

- tplot=binary output plot file containing the thermal response results that can be graphically displayed using the post processor ORION [7]. The default root name is " $t 2 p l o t$ ". 
- $\quad \mathrm{vff}=$ binary input file containing view factors calculated using the code FACET [8] or exchange factors calculated using the code MONTE2D [9]. The default name is "fabs".

- $\mathrm{dpf}=$ binary output file containing information necessary to restart CAST2D. This is a dump file with the default root name "c2dump".

- $\mathrm{rtf}=$ binary input file containing information necessary to restart CAST2D. This is a dump file from a previous run.

File name dropouts are permitted, for example

\section{cast2d $i=i n f$}

\subsection{Interactive Controls}

Several interactive controls are available to the user when executing CAST2D. The controls allow the user to query the status of the code execution or to terminate execution. The interrupt commands and their resulting system response are described in the following list.

sw1. a dump file is written and CAST2D terminates

sw2. CAST2D responds with time, cycle number, and solution convergence information for the mechanical calculation at the current time step

sw3. CAST2D responds with time, cycle number, and solution convergence information for the mechanical calculations for each time step until sw3. is retyped

sw4. a dump file is written and CAST2D continues execution

sw5. CAST2D responds with time, cycle number, and solution convergence information for the thermal calculation at the current time step

sw6. CAST2D responds with time, cycle number, and solution convergence information for the thermal calculations for each time step until sw6. is retyped

On workstations (e.g., SUN), first type control-c for a system interrupt and then type the above commands for the desired action. 


\section{References}

1 A. B. Shapiro, "TOPAZ2D - A Two Dimensional Finite Element Code for Heat "Transfer Analysis, Electrostatics, and Magnetostatic Problems", University of California, Lawrence Livermore National Laboratory, Rept

UCID-20824, 1986.

2 J. O. Hallquist, "NIKE2D - A Vectorized Implicit, Finite Deformation, Finite Element Code for Analyzing the Static and Dynamic Response of 2-D Solids with Interactive Rezoning and Graphics", University of California, Lawrence Livermore National Laboratory, Rept UCID$19677,1986$.

3 ProCAST - The Professional Casting Simulation Tool, UES Inc., Dayton, OH, 1991.

4 M.A. Gerhard, private communication, 1984.

5 E.C. Flower,"Non-isothermal FEM Analyses of Large-Strain Back Extrusion Forging",University of California, Lawrence Livermore National Laboratory, Rept UCRL-94691, June 1986.

6 J.O. Hallquist, "MAZE - An Input Generator for DYNA2D, NIKE2D, and TOPAZ2D", University of California, Lawrence Livermore National Laboratory, Rept UCID-19029, June 1983.

7 J. O. Hallquist, "ORION - An Interactive Color Post processor for 2-Dimensional Finite Element Codes", University of California, Lawrence Livermore National Laboratory, Rept UCID-19310, 1985.

8 A. B. Shapiro, "FACET - A Radiation View Factor Computer Code for Axisymmetric, 2D Planar, and 3D Geometries with Shadowing", University of California, Lawrence Livermore National Laboratory, Rept UCID-19887, 1983. 
9 P.J. Burns, "MONTE - A Two-dimensional Radiative Exchange Factor Code", Colorado State University, Ft. Collins, CO (1983). 

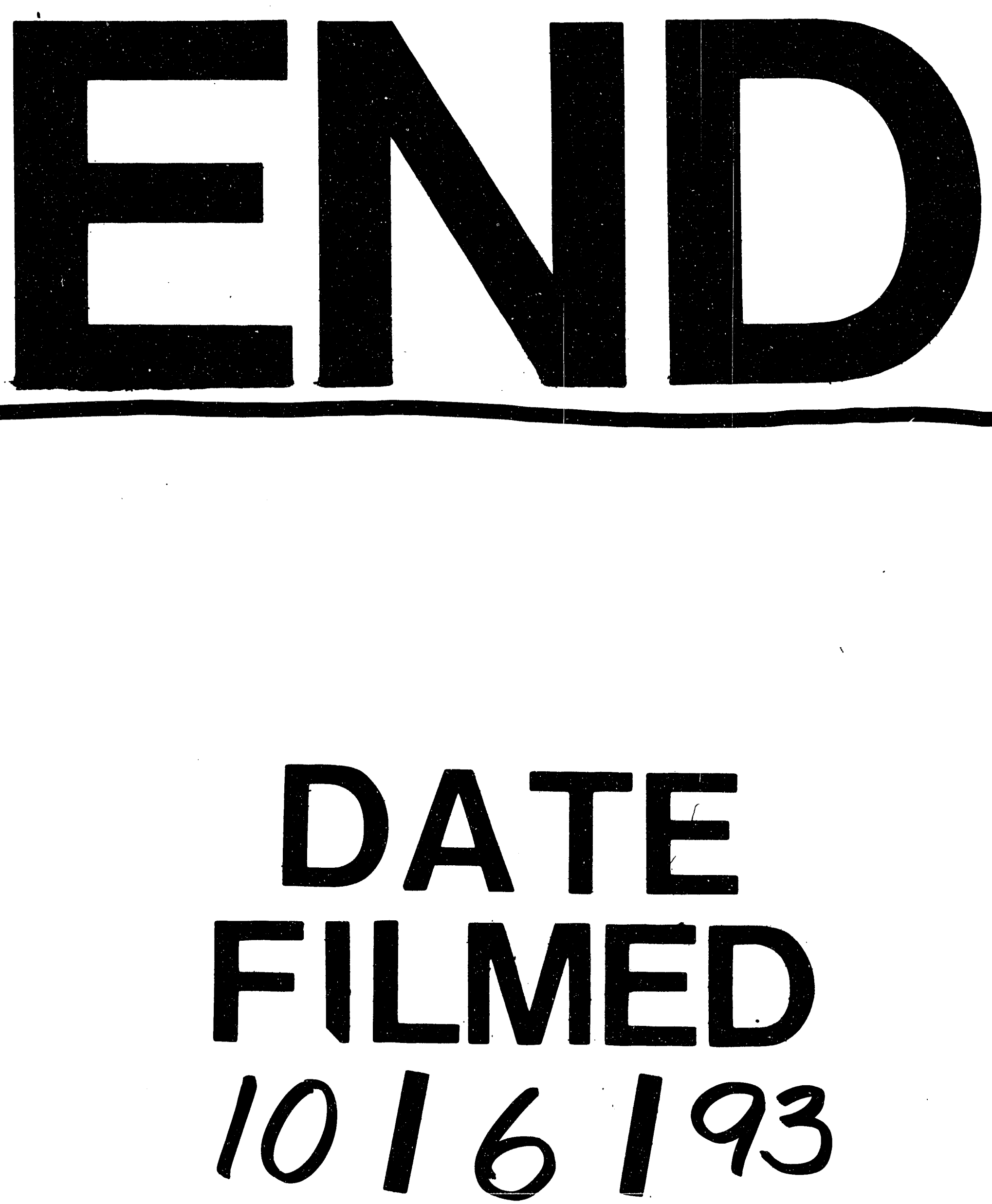
